\title{
A mobilidade independente da criança em centros urbanos
}

The child's independent mobility in urban centers

Mobilité indépendante de l'enfant en centres urbains

Movilidad independiente del niño en los centros urbanos

\author{
Gabriela Mello Sabbag* \\ (gabi_s@hotmail.com) \\ Ariane Kuhnen** \\ (ariane.kuhnen@ufsc.br) \\ Mauro Luís Vieira** \\ (maurolvieira@gmail.com)
}

Recebido em 23/11/2013; revisado e aprovado em 14/07/2014; aceito em 28/12/2014

DOI: http://dx.doi.org/10.1590/1518-70122015217

\begin{abstract}
Resumo: A diminuição da mobilidade infantil independente, em grandes centros urbanos, vem sendo detectada e relacionada a fatores como a violência e a falta de espaços apropriados para as crianças. Por meio de revisão bibliográfica internacional, foi identificado que a mobilidade estimula o desenvolvimento cognitivo, social e motor da criança e que há necessidade de políticas e planejamentos urbanos que proporcionem espaços seguros e maior participação da criança na cidade.

Palavras-chave: Mobilidade independente. Criança. Urbano.
\end{abstract}

Abstract: Decreased of mobility independent children in large urban centers has been detected and related to factors such as violence and lack of appropriate spaces for children. Through international literature review, it was identified that mobility stimulates cognitive, social and motor child development and that there is need for policy and urban planning that provide safe spaces and greater participation of the child in the city.

Key words: Independent mobility. Child. Urban.

Résumé: Les diminution de la mobilité des enfants indépendants dans les grands centres urbains, a été détectee et liée à des facteurs tels que la violence et le manque d'espaces appropriés pour les enfants. Grâce à l'examen de la littérature internationale, il a été identifié que la mobilité stimule cognitif, social et moteur de l'enfant et qu'il est nécessaire pour la politique et la planification urbaine qui fournissent des espaces sûrs et une plus grande participation de l'enfant dans la ville.

Mots-clés: La mobilité individuelle. Enfants. Urbain.

Resumen: La disminución de la movilidad hijos independientes en los grandes centros urbanos, se ha detectado y en relación con factores como la violencia y la falta de espacios adecuados para los niños. Mediante revisión de la literatura internacional, se identificó que la movilidad estimula el desarrollo cognitivo, social y motor del niño y que hay necesidad de que la política y la planificación urbana que proporcionan espacios seguros y una mayor participación de los niños en la ciudad.

Palabras clave: La movilidad independiente. Los niños. Urbano.

\section{Introdução}

Pesquisadores vêm demonstrando preocupação com a qualidade do espaço urbano que vem sendo ofertado para as crianças interagirem e explorarem o ambiente de maneira independente. Na Europa, tal tema vem sendo debatido desde 1970 (VISSCHER; BIE, 2008), segundo Hillman e Adams (1992), o nível de liberdade de locomoção independente da criança, entre os anos de 1971 e 1990, diminuiu, e o principal fator associado ao decréscimo da liberdade das crianças é o medo do tráfego por parte dos pais. Há uma preocupação com a segurança do público infantil em centros urbanos (BJÖRKLID, 1994; CRUZ, 2011), mas também com as crianças superprotegidas, muito controladas, isoladas, pouco ativas e independentes em seus ambientes (KYTTÄ, 1997). Investigações sobre a mobilidade e a saúde da criança, os ambientes facilitadores das atividades físicas e sua relação com o desenvolvimento físico e motor da criança e do adolescente, vêm sendo realizadas (CARVER et al., 2010; CHRISTENSEN et al., 2011; DAVIS; JONES, 1996; NETO; MALHO, 2004).

\footnotetext{
* Faculdade de Administração, Ciências, Educação e Letras (FACEL), Curitiba, Paraná, Brasil.

** Universidade Federal de Santa Catarina, Florianópolis, Santa Catarina, Brasil.
} 
Alguns questionamentos surgem sobre essa temática: Como as crianças fazem para se locomover em seu dia a dia? Como vão para a escola? Visitam amigos? Brincam sozinhas, com amigos ou sempre acompanhadas por adultos? Em quais ambientes físicos as crianças brincam (internos e/ou externos)? Quais as atividades das crianças em ambientes internos e externos? O quanto uma criança pode exercer a independência numa cidade? As crianças têm oportunidades para interagir entre si ou sempre são intermediadas por adultos? Quais os efeitos das práticas de restrição do uso de espaços externos para o seu desenvolvimento físico, motor, cognitivo e social da criança? (PREZZA et al., 2001; O'BRIEN et al., 2000; KYTTÄ, 1997; NETO; MALHO, 2004).

Kyttä (1997) e Neto e Malho (2004) citam que o desenvolvimento da criança não pode se restringir ao espaço da sua casa, ao quintal e ao playground, sendo que há uma vizinhança, um bairro ao redor com possibilidades de interações (affordances) a serem estabelecidas pela criança. No entanto, quando essas oportunidades são impedidas por barreiras físicas (DAVIS; JONES, 1996; MOUETTE; AIDAR; WAISMAN, 2000; CRUZ, 2011) e sociais (VALENTINE; MCKENDRICK, 1997), efeitos tais como a diminuição de repertório social, motor e de conhecimento de atividades lúdicas e cotidianas vêm sendo detectados (KYTTÄ, 2004; VISSCHER; BIE, 2008; PREZZA et al., 2001). Apesar da detecção de que há barreiras que limitam a mobilidade da criança em ambientes urbanos, muitas vezes estas são desconsideradas no planejamento das cidades e nas políticas públicas (DAVIS; JONES, 1996).

Para analisar a mobilidade e suas relações com o desenvolvimento infantil, deve-se considerar: o espaço urbano disponível (PREZZA et al., 2001; CRUZ, 2011), as práticas sociais e culturais relacionadas as práticas parentais (O'BRIEN et al., 2000; POOLEY; TURNBULL; ADAMS, 2005; PREZZA et al., 2005), as condições de vida e de moradia das famílias (CRUZ, 2011; PREZZA et al., 2001), características como a idade e o gênero (POOLEY; TURNBULL; ADAMS, 2005; PREZZA et al., 2001) e as habilidades da criança (VILLANUEVA et al., 2012).

Diante desses fatores, pretende-se apresentar as contribuições das pesquisas da área, em especial as internacionais, sobre o processo de desenvolvimento da mobilidade da criança ocidental em centros urbanos, buscando analisar: a relação entre a mobilidade e o desenvolvimento humano; a perspectiva de desenvolvimento infantil e a mobilidade; a mobilidade e a independência infantil em centros urbanos e; o método e os resultados de algumas pesquisas sobre mobilidade e independência infantil.

\section{Mobilidade e desenvolvimento humano}

Günther (2003), ao definir mobilidade, destaca a necessidade de se considerar as relações recíprocas entre a pessoa que se comporta e o ambiente físico, sendo que a mobilidade possibilita a ocorrência dessas interações. O autor destaca que, conforme a pessoa se movimenta e se desloca num espaço físico, ela o modifica (tanto em termos físicos, quanto sociais), assim como esse ambiente físico possibilita ou não determinados deslocamentos e ações por parte da pessoa. Os comportamentos de movimentação, locomoção e exploração do ambiente estão relacionados aos contextos sociais que podem favorecê-los ou não, tais como: os aspectos socioculturais, percebidos ou não pela pessoa, sobre o espaço pessoal e a privacidade (GÜNTHER, 2003). Apesar da variedade de contextos sociais e culturais que envolvem o ser humano, os autores (KYTTÄ, 2004; GÜNTHER, 2003) enfatizam que a mobilidade no ambiente físico, enquanto exploração é essencial para o desenvolvimento. Ao longo do desenvolvimento humano, surgem novas demandas e contextos cada vez mais complexos, os quais exigem o contato e a exploração de recursos do ambiente físico e social, desde a infância até a fase adulta (CRUZ, 2011; GÜNTHER, 2003; NETO; MALHO, 2004). Quando há eventos que impedem a mobilidade, como as restrições a nível pessoal ou ambiental, podem ocorrer interferências que afetam o percurso do desenvolvimento (GÜNTHER, 2003).

A mobilidade diária traz implicações para a construção da vida cotidiana, consequentemente, ocasiona mudanças e possibilita a construção da identidade individual e grupal (POOLEY; TURNBULL; ADAMS, 2005). Para a caracterização da mobilidade, deve-se 
buscar investigar: para onde a pessoa vai (residência, escola, emprego, casa de amigos ou familiares, hospital, parques, ruas); como ela transita (a pé, de bicicleta, de skate, patins, de carro, de ônibus, de metrô, trem, avião); com quem ela se locomove (acompanhada ou desacompanhada); para que ela se movimenta e com que frequência ela o faz. Esses fatores trazem implicações para a identidade, consequentemente, para o desenvolvimento de uma pessoa (POOLEY; TURNBULL; ADAMS, 2005).

Além disso, há dimensões que devem ser consideradas e que, quando combinadas, explicam o processo da mobilidade e suas mudanças ao longo do tempo: idade, gênero, grupo socioeconômico, estrutura familiar, estrutura urbana, a tecnologia para o transporte e a rede de transporte que existem em determinadas localidades (POOLEY; TURNBULL; ADAMS, 2005).

Sobre a mobilidade infantil, Neto e Malho (2004) citam que as ações e as interações vivenciadas pela criança em diversos contextos são fundamentais para o desenvolvimento e a organização dos processos adaptativos e competências para a interação social dinâmica.

\section{Perspectivas sobre o desenvolvimento infantil e a mobilidade}

Os estudiosos do tema mobilidade, frequentemente, argumentam que há uma relação entre a visão que se tem da criança e as práticas que possibilitam maior liberdade e movimentação das crianças nas cidades (VISSCHER; BIE, 2008; DAVIS; JONES, 1996; NETO; MALHO, 2004; KYTT ̈̈, 2004; CHRISTENSEN et al., 2011). Reflexões baseadas nos estudos sobre a sociologia da criança (ARIÉS, 1981) propõem que as crianças sejam consideradas como seres humanos com direitos, necessidades e que lhes devam ser ofertadas possibilidades para que sejam ativas em seus contextos de interação. Essas propostas influenciam as pesquisas sobre mobilidade e a independência da criança, as quais afirmam que esta não deve ser percebida como um ser que sempre precisa se adequar às necessidades e aos parâmetros impostos pelos adultos (VISSCHER; BIE, 2008). Os estudiosos do tema destacam que, muitas vezes, as crianças são vistas como seres que ainda não desenvolveram o raciocínio e que, por essa razão, precisam ser contidos e controlados pelos adultos sempre (DAVIS; JONES, 1996; NETO; MALHO, 2004; KYTTÄ, 2004; CHRISTENSEN et al., 2011), fato que pode estar relacionado às restrições à liberdade infantil. Segundo Davi e Jones (1996), os estudos sobre transporte não propõem restrições aos veículos, mas sim às crianças, que devem ser controladas ativamente pelos responsáveis, sendo impostas barreiras que podem ocasionar consequências para o desenvolvimento infantil que vêm preocupando os pesquisadores, tais como: a diminuição da autonomia (KYTTÄ, 2004), o sentimento de medo constante (BJÖRKLID, 1994), os atrasos no desenvolvimento psicomotor e as poucas oportunidades de interação social em espaços físicos mais livres, como a rua (NETO; MALHO, 2004).

Visscher e Bie (2008) afirmam que há duas perspectivas sobre o uso do espaço público urbano pelas crianças e que elas afetam as estratégias de educação e socialização. A primeira propõe que a criança seja protegida dos perigos ofertados pela cidade, o que resulta em segregação e separação dos espaços frequentados, ficando a criança restrita a maior parte do tempo aos ambientes institucionalizados: escola, casa e instituições recreacionais. A segunda argumenta que a criança deve ser preparada e estimulada para se tornar capaz de se mover, independentemente e de forma segura, nos espaços públicos urbanos, podendo acessar as oportunidades de socialização ofertadas pela cidade, tornando-se "amiga da cidade" (usuária e conhecedora dos recursos de uma cidade).

A perspectiva de controle ativo da vida da criança está associada à institucionalização infantil, termo que tem sido utilizado para se referir a atividades e espaços pedagógicos por ela frequentados, diariamente e por muitas horas, em que há atividades estruturadas, um controle ativo do desenvolvimento da criança, o que pode ser considerado como um preparo da criança para o seu futuro na sociedade. Nem sempre essa proposta considera a socialização nos espaços públicos como educativa e necessária para o desenvolvimento infantil (VISSCHER; BIE, 2008).

$\mathrm{Na}$ realidade europeia, as estratégias utilizadas para estimular o uso dos ambientes 
públicos em cidades por crianças vêm passando por transformações. Visscher e Bie (2008) citam que, no final do século XIX, surgiu o playground tradicional (espaço externo organizado para as crianças brincarem) com o objetivo de que as crianças de ambientes urbanos pudessem usufruir espaços externos, mas que este falhou no quesito socialização. A partir de então, século XX, novos estudos buscaram analisar novas propostas de espaços externos para as crianças menos formais, mais livres que possibilitassem a interação entre as crianças e as habilidades de criatividade (VISSCHER; BIE, 2008).

Em meados da década de 1990 e início dos anos 2000, na Europa (Holanda, Bélgica), surgiram propostas de mudança do design dos bairros para buscar incluir as crianças e promover maior interação independente destas em centros urbanos. O novo modelo foi denominado play web, este é formado por redes integradas por meio da construção de rotas seguras que dão acesso aos parques, playgrounds, escolas, centros de esporte e, nessas rotas seguras, as crianças podem se locomover de maneira independente em seus bairros. Tais iniciativas fazem parte do programa Child-friendly-city, cidade amigável para criança, uma proposta política, introduzida pela The United Nations Children's Fund (UNICEF, 2013) em 1996, que tem o objetivo de ofertar à criança o direito de andar em segurança, encontrar os amigos, interagir e brincar por conta própria, participar ativamente da vida comunitária, bem como expressar suas opiniões sobre a vida na cidade (VISSCHER; BIE, 2008; UNICEF, 2013). A perspectiva da criança como um ser ativo busca ofertar o direito a acessibilidade, flexibilidade, segurança e oportunidades de interação, fatores que estão relacionados às possibilidades de mobilidade independente (BLINKERT, 2004).

\section{Mobilidade e independência da criança}

A definição de independência de mobilidade envolve a análise do desenvolvimento da autonomia da criança e a consideração das possibilidades ofertadas pelos espaços físicos acessíveis a mesma (NETO; MALHO, 2004). Sendo a independência uma capacidade que emerge das oportunidades e experiências que a criança tem para tomar decisões de se movimentar num espaço físico, o qual oferta possibilidades de ação para a criança (NETO; MALHO, 2004).

A independência de mobilidade é conquistada gradativamente, depende do desenvolvimento maturacional da criança, mas também das experiências que ela vivencia em seus contextos de interação (NETO; MALHO, 2004). Um estudo brasileiro, desenvolvido por Cruz (2011), detectou a forma como as crianças organizavam e estruturavam a percepção e a sua atuação em contextos cada vez mais complexos. Cruz (2011) observou que as crianças diariamente, por meio das experiências com o meio, iam organizando o espaço e, de acordo com a evolução da idade, da complexidade das atividades e dos contextos, iam modificando sua interação. Elas iam obtendo maior conhecimento e consciência, inicialmente, da casa em seu ambiente interior e dos membros que faziam parte desta; posteriormente, da casa como um todo e um ambiente geográfico interno, na fase mais avançada passavam a compreender a casa e os contextos externos, tais como o quintal e o terreno, para futuramente localizar a casa numa rua e as ruas mais próximas a esta (HIGUCHI, 1999, citado em CRUZ, 2011).

Desta forma, a mobilidade independente deve ser analisada de uma perspectiva evolutiva em que conforme a criança cresce e tem oportunidades de exploração dos espaços, ela vai desenvolvendo representações destes espaços físicos (memória, percepção e identificação), o que possibilita a liberdade de ação e as novas interações com os espaços cotidianos (NETO; MALHO, 2004).

Sobre as estratégias para medir e analisar a mobilidade independente infantil, Kyttä (2004) explica que a independência pode ser analisada do ponto de vista do alcance territorial das crianças, isto é, a distância percorrida pela criança entre a residência e os locais que ela frequenta, estando ela sozinha ou com pares. Neto e Malho (2004) propõem que se busque descobrir: a maior distância percorrida pela criança; se ela realiza os trajetos sozinha e/ ou com amigos de idade aproximada, mais jovens ou mais velhos; a análise dos trajetos e caminhos realizados pela criança de sua casa para a escola; o meio de transporte utilizado no percurso; a motivação das crianças para 
frequentarem ou não os locais públicos; o fato de frequentarem ou não a casa de amigos e/ ou vizinhos do bairro.

Para a análise da mobilidade infantil, também deve ser considerada a licença ou permissão que a criança tem para se mover de forma independente no ambiente, sendo que o nível de mobilidade está relacionado às regras definidas pelos pais/ cuidadores (KYTTÄ, 2004; CARVER; TIMPERIO; CRAWFORD 2012; O'BRIEN et al., 2000). Os autores destacam o papel dos familiares que podem facilitar ou restringir as oportunidades de exploração do ambiente físico pela criança (HILLMAN; ADAMS, 1992; NETO; MALHO, 2004; KYTTÄ, 2004). Mas além desse fator, no que se refere à mobilidade infantil nas cidades, as pesquisas (DAVIS; JONES, 1996; MOUETTE; AIDAR; WAISMAN, 2000; KYTTÄ, 2004) apontam para as mudanças dos espaços físicos (calçadas perigosas, ruas sem calçadas, falta de espaço próprio para as crianças brincarem) e para os altos índices de violência e envolvimento de crianças e adolescentes com crimes (VALENTINE; MCKENDRICK, 1997), como variáveis que dificultam a possibilidade de os pais ofertarem liberdade para as crianças interagirem com o espaço urbano de forma mais livre e independente. Neto (1999) cita que os espaços públicos das cidades, em especial as ruas, vêm deixando de ser locais que propiciam oportunidade para interação com outras crianças ou com outras gerações, seja para brincar ou para passar um tempo livre e espontâneo.

\section{Métodos e resultados dos estudos sobre mobilidade da criança em centros urbanos}

Para estudar a mobilidade infantil em contextos urbanos, os pesquisadores utilizam estratégias metodológicas variadas e múltiplas que visam detectar a interação entre os fatores associados, sendo utilizados recursos, tais como: entrevistas e/ou questionários aplicados às crianças e aos pais/cuidadores (KYTTÄ, 1997); observações sistemáticas do comportamento de deslocamento infantil (CHRISTENSEN et al., 2011); exercícios de mapas, nos quais os pesquisadores buscam detectar os trajetos das crianças (O'BRIEN et al., 2000); observações e fotos da vizinhança; utilização de recursos tecnológicos, tais como o uso do Global Positioning System (GPS), combinado com o trabalho de campo etnográfico e um questionário interativo a que a criança responde via telefone móvel enquanto se locomove no seu dia a dia (CHRISTENSEN et al., 2011); utilização de diários sobre os trajetos/ deslocamentos em que a criança deve descrever se transita acompanhada ou não, o meio de transporte utilizado e o significado das experiências de mobilidade (KYTTÄ, 1997); análise histórica e documental de implantações de propostas políticas e pedagógicas em algumas cidades europeias (VISSCHER; BIE, 2008); análise da participação das crianças nas políticas públicas de saúde (DAVIS; JONES, 1996; RISOTTO et al., 2008).

Nas entrevistas com as crianças, são explorados temas como: o que elas pensam sobre os locais; quais espaços são significativos; o que elas fazem nesses locais; os espaços que utilizam para brincar, andar, correr; como exploram os ambientes, se tem liberdade para se locomover sozinhas ou com colegas; mapeamento dos locais que frequentam e trajetos; a supervisão e prática regulatória parental (O'BRIEN et al., 2000). Por meio das observações sistemáticas, busca-se descrever todas as atividades realizadas pelas crianças. Os pesquisadores obtêm permissão, dos pais e da escola, para ficar na sala de aula durante as atividades escolares, no recreio quando as crianças brincam lá fora, após a saída da escola, quando as crianças aproveitam o tempo em casa e na comunidade local e aqueles fazem perguntas ou tiram dúvidas das crianças sobre como utilizar os instrumentos da pesquisa (CHRISTENSEN et al., 2011).

Por meio desses procedimentos, as pesquisas vêm detectando a diminuição da mobilidade independente relacionadas a diversos fatores que se inter-relacionam: práticas parentais, ambientes físicos e contextos sociais das cidades, as características (idade, gênero) e o repertório da criança (habilidades).

Sobre as práticas familiares, $\mathrm{O}^{\prime}$ Brien et al. (2000) destacam que não somente as práticas parentais devem ser consideradas para a compreensão da mobilidade infantil, mas também a cultura familiar, as transmissões de informações entre as gerações sobre as possibilidades de acesso infantil aos ambientes públicos, bem como o repertório da criança. As ações parentais na interação com os filhos 
não ocorrem desconectadas de fatores sociais e ambientais, quando os pais consideram os ambientes como perigosos, devido ao intenso tráfego de carros (HILLMAN; ADAMS, 1992), às ruas pouco seguras (VILLANUEVA et al., 2012), aos riscos do contato com estranhos, à possibilidade de sequestros ou de envolvimento da criança em situações criminosas (VALENTINE; MCKENDRICK, 1997; RODRIGUES; PESSOA; SILVA, 2007), esses fatores aumentam as restrições à mobilidade infantil. As crianças não podem ir para a escola desacompanhadas de adultos (HILLMAN; ADAMS, 1992; RODRIGUES; PESSOA; SILVA, 2007), poucas vão para a escola a pé (MALHO, 2004), os pais passam a levar a criança para a escola de carro (CARVER; TIMPERIO; CRAWFORD, 2012; MALHO, 2004), havendo um isolamento da criança e a supervisão parental constante (BJÖRKLID, 1994). Segundo Malho (2004), as experiências infantis no espaço urbano, geralmente, são mediadas pelos adultos ou por meios que afastam a criança da experiência real e corporal nesses ambientes, como quando estão dentro de automóveis. Dados contrários também são encontrados: as percepções de pais e filhos sobre as rodovias e ruas como sendo seguras estão associadas à maior frequência de atividades físicas por crianças e jovens (de 8 a 9 anos e 13 a 15 anos) (CARVER et al., 2010), assim como as mães que se sentem pertencentes à comunidade, as quais conhecem e se relacionam com seus vizinhos, permitem que seus filhos transitem pelo bairro (PREZZA et al., 2001).

Sobre o espaço urbano disponibilizado para as crianças, também devem ser analisadas a localização, as características da moradia, a disponibilidade ou a ausência de parques e de centros públicos de lazer na região da residência (PREZZA et al., 2001; CRUZ, 2011). Na pesquisa de O'Brien et al (2000), as crianças que residiam em locais próximos a parques e de fácil acesso aos ambientes de lazer (clube, casa dos amigos) se locomoviam mais livremente e realizavam mais atividades ao ar livre, iam brincar no parque e na piscina do clube. Num centro urbano da Austrália, os locais mais frequentados pelas crianças foram as escolas, as casas dos amigos, as lojas, os parques (VILLANUEVA et al., 2012). Já num centro urbano brasileiro, as principais ativi- dades realizadas pelas crianças envolveram o estudar, o fazer tarefas de casa e o brincar em casa (RODRIGUES; PESSOA; SILVA, 2007). Sobre as atividades e os contextos de crianças brasileiras que residiam numa zona de ocupação de Manaus (CRUZ, 2011), foi detectado que as crianças precisavam de espaço para se movimentar, para desenvolver a motricidade e que acabavam brincando em locais que ofereciam perigos como o beco, no qual passavam muitas pessoas e até mesmo motos, havia risco de as crianças deslizarem, sendo que foram relatados acidentes como atropelamentos, mas também as estratégias que as crianças desenvolveram para brincar e explorar o ambiente, o que lhs aumentava a autonomia. No entanto Cruz (2011) destacou que, na realidade brasileira, a maioria das cidades apresentam problemas sociais e ambientais que dificultam a mobilidade infantil, tais como aglomeração, pobreza, periferias geográficas e sociais, desemprego, depredação dos recursos naturais e falta de infraestrutura para o saneamento básico e para a segurança.

Outro achado foi que as crianças residentes em cidades pequenas ou vilas, geralmente, apresentam maior mobilidade independente que as crianças de áreas urbanas e rurais (CHRISTENSEN et al., 2011; CARVER; TIMPERIO; CRAWFORD, 2012). Há também diferenças no nível de mobilidade a depender do país em que a criança reside, Kyttä (1997; 2004) detectou que as crianças finlandesas apresentam maiores níveis de liberdade de locomoção que as crianças do Reino Unido, Alemanha e Austrália. Tais diferenças estão relacionadas às diferenças culturais, ao senso de comunidade, bem como à organização e espaços públicos disponibilizados (KYTTÄ, 1997; PREZZA, 2001).

Sobre a relação entre as características da criança e a mobilidade, ocorre o aumento gradativo da liberdade de mobilidade associado ao aumento da idade da criança (O'BRIEN et al., 2000; PREZZA et al., 2001) e ao gênero masculino (PREZZA et al., 2001; KYTTÄ, 2004). No entanto, de maneira geral, as crianças já não têm tantas possibilidades para realizar atividades sozinhas como antigamente (KYTTÄ, 2004; HILLMAN; ADAMS, 1992).

Sobre o repertório das crianças, o nível de mobilidade infantil influencia o desenvolvimento físico, emocional, cognitivo e social 
da criança (KYTTÄ, 2004). As crianças que têm oportunidade de brincar fora de casa apresentam: maior frequência de interações entre pares (seja em ambientes internos ou externos), mais comportamentos de exploração do espaço físico, senso de responsabilidade e de cuidado do ambiente físico (KYTTÄ, 2004; NETTO; MALHO, 2004); maior conhecimento das atividades lúdicas (KYTTÄ, 1997); do que as crianças que só brincam em playgrounds.

Por fim, alguns estudos apresentam propostas políticas e pedagógicas alternativas para que a criança possa usufruir dos centros urbanos com maior autonomia e liberdade. Davis e Jones (1996) afirmam que o planejamento da cidade e o uso desta pelas crianças é um tema de política pública de saúde, mas que mudanças voltadas para o bem-estar da criança estão relacionadas a outros setores da sociedade, como a economia. Sobre a área econômica, os meios de transporte envolvem um mercado que traz lucros e benefícios financeiros para a sociedade. Já a criança e suas necessidades para o desenvolvimento saudável em contextos urbanos, geralmente, são lembradas somente quando há problemas da infância e juventude. Davis e Jones (1996) citam que essa população, frequentemente, é vista como vítima ou problemática, o que dificulta a busca de soluções que valorizem e possibilitem o desenvolvimento de capacidades, incluindo a autonomia e a liberdade. Baseados na visão de saúde da Organização Mundial da Saúde, os autores destacam que o desenvolvimento saudável da criança em ambientes urbanos deve proporcionar oportunidades para que ela possa utilizar a cidade, brincar e se locomover nas ruas com certo grau de independência, tomar algumas decisões e ser ouvida. Risotto et al. (2008) propõem a inovação e a experimentação de serviços socioeducativos para crianças e adolescentes, serviços de preparo e suporte para pais e medidas de implementação que melhorem o acesso e uso dos ambientes urbanos pelas minorias e promoção dos direitos e da cidadania das crianças. As autoras propõem que ações territoriais sejam realizadas por meio da detecção das necessidades das famílias e das crianças e o estabelecimento de metas prioritárias. Estas estão em consonância com as políticas públicas que buscam contar com a participação ativa da comunidade para a tomada de decisão referente à saúde pública.

\section{Considerações finais}

A mobilidade acompanha o ser humano ao longo de sua trajetória, sendo aumentada ou diminuída de acordo com a fase e o contexto de vida da pessoa. O surgimento dos grandes centros urbanos trouxeram mudanças para o estilo de vida e a rotina das pessoas, consequentemente, para a mobilidade.

No caso da mobilidade infantil, o tema vem sendo pesquisado em países europeus, os quais vêm detectando a diminuição da liberdade e da mobilidade independente das crianças e seus efeitos para o desenvolvimento infantil, tais como a diminuição da autonomia, do livre trânsito e a dificuldade de reconhecimento do espaço geográfico da cidade pela criança. São sugeridas intervenções ambientais e mudanças no design nas cidades que possibilitem maior segurança, menor tráfego e facilidade para chegar aos destinos, construção de rotas mais seguras e locais que sejam atrativos e confortáveis para as crianças. Ao mesmo tempo, programas educativos para o desenvolvimento de habilidades infantis para transitarem em segurança na vizinhança voltados para os pais e para as crianças. Há propostas para que as crianças sejam incentivadas a participar das políticas públicas de saúde e planejamento urbano de forma ativa.

Para a elaboração de propostas e novas políticas relacionadas à mobilidade em centros urbanos é preciso destacar que esta é uma área de que merece a análise da perspectiva de várias disciplinas: engenharia, arquitetura, psicologia, educação física, medicina, educação, fisioterapia, dentre outros setores.

Há poucos estudos brasileiros sobre esta temática, sendo que o Brasil é um país que contém grandes centros urbanos com diferenças geográficas, espaciais e sociais relevantes. Com isso, surgem questionamentos sobre como as crianças brasileiras de diferentes regiões, cidades e contextos (centrais, periféricos) estão vivenciando a sua infância na cidade. Como as políticas públicas nacionais analisam a situação da criança nos centros urbanos brasileiros? Como os pais brasileiros analisam e direcionam a mobilidade de seus filhos? Como as crianças brasileiras percebem sua liberdade de mobilidade? Quais têm sido as consequências dessas práticas para a o desenvolvimento das crianças brasileiras? Essas 
perguntas envolvem temas que podem ser investigados por pesquisadores, os quais podem buscar o acesso à realidade das crianças de diferentes contextos geográficos brasileiros, visando descrever as possibilidades de mobilidade independente das crianças no Brasil.

\section{Referências}

ARIÉS, Philipe. História social da criança e da família. Rio de Janeiro: Zahar, 1981.

BJÖRKLID, Pia. Children, traffic, environment. Architecture and Behaviour, v. 10, n. 4, p. 399-406, 1994.

BLINKERT, B. Quality of the city for children: chaos and order. Children, youth and environments, v. 14, n. 2, p. 99-112, 2004.

CARVER, Alison; TIMPERIO, Ana; HESKETH, Kylie; CRAWFORD, David. Are safety-related features of the road environment associated with smaller declines in physical activity among youth? Journal of urban health: Bulletin of the new york academy of medicine, v. 87, n. 1, p. 29-43, 2010.

CARVER, Alison; TIMPERIO, Ana F.; CRAWFORD, David A. Young and free? A study of independent mobility among urban and rural dwelling Australian children. Journal of science and medicine in sport, n. 15, p. 505-510, 2012.

CHRISTENSEN, Pia; MIKKELSEN, Miguel Romero; NIELSEN, Thomas Alexander Sick; HARDER, Henrik. Children, mobility, and space: using GPS and mobile phone technologies in ethnographic research. Journal of Mixed Methods Research, p.1-20, 2011.

CRUZ, Patrícia de Goes. Ambiente urbano: lugar de restrição espacial e descoberta de novos espaços. Saúde Soc., São Paulo, v. 20, n. 3, p. 702-714, 2011.

DAVIS, Adrian; JONES, Linda L. Children in the urban environment: an issue for the new publice health agenda. Health \& Place, v. 2, n. 2, p. 107-113, 1996.

GÜNTHER, Hartmut. Mobilidade e affordance como cerne dos estudos pessoa-ambiente. Estudos de Psicologia, v. 8, n. 2, p. 273-280, 2003.

HILLMAN, Mayer; ADAMS, John G.U. Children's freedom and safety. Children's Environments, v. 9, n. 2, p. 12-33, 1992.

KYTTÄ, Marketta. Children's independent mobility in urban, small town, and rural environments. In: CAMSTRA (Org.). Growing up in a changing urban landscape. Assen: Van Gorcum, 1997. p. 41-52.

. The extent of children's independent mobility and the number of actualized affordances as criteria for child-friendly environments. Journal of Environmental Psychology, n. 24, p. 179-198, 2004.

MALHO, Maria João. A criança e a cidade: independência de mobilidade e representações sobre o espaço urbano. Actas dos ateliers do $V^{o}$ congresso português de sociologia, v. 5, p. 49-56, 2004. Disponível em: <http:/ / www.aps. pt/cms/docs_prv/docs/DPR4628ed529c42d_1.pdf>. Acesso em: 12 ago. 2013.

MOUETTE, Dominique; AIDAR, Tirza; WAISMAN, Jaime. Avaliação dos impactos do tráfego na mobilidade da população infantil através da análise de correspondência múltipla, Transportes, v. 8, n. 1, p. 56-87, 2000.

NETO, Carlos. O jogo e os quotidianos de vida da criança. In: KREBS, Ruy; COPETTI, Fernando; BELTRAME, Tais S.; USTRA, Marcelo (Org.). Perspectivas para o desenvolvimento infantil. Santa Maria, RS: SIEC, 1999. p. 49-66.

NETO, Carlos; MALHO, Maria João. Espaço urbano e independência de mobilidade na infância. Boletim do IAC, Lisboa, n. 73, separata n. 11, p. 1-4, 2004. Disponível em: <http://www.fmh.utl.pt/Cmotricidade/dm/ textoscn/espacourbano.pdf> Acesso em: 12 ago. 2013.

O'BRIEN, Margaret; JONES, Deborah; SLOAN, David; RUSTIN, Michael. Children's independent spatial mobility in the urban public realm. Childhood, v. 7, v. 3, p. 257-277, 2000.

POOLEY, Colin.G.; TURNBULL, Jean; ADAMS, Mags. The Significance of travel and mobility. In: POOLEY, Colin.G.; TURNBULL, Jean; ADAMS, Mags (Org.). A mobile century: changes in everyday mobility in Britain in the twentieth century. Aldershot: Ashgate, p. 1-12, 2005.

PREZZA, Miretta; ALPARONE, Francesca R.; CRISTALLO, Carmela; LUIGI, Secchiano. Parental perception of social risk and of positive potentiality of outdoor autonomy for children: the development of two instruments. Journal of environmental psychology, n. 25, p. 437-453, 2005.

PREZZA, Miretta; PILLONI, Stefania; MORABITO, Carmela; SERSANTE, Cinzia; ALPARONE, Francesca R.; GIULIANI, Maria V. The influence of psychosocial and environmental factors on children's independent mobility and relationship to peer frequentation. Journal of community $\mathcal{E}$ applied social psychology, v. 11, n. 6, p. 435-450, 2001.

RISOTTO, Antonella; ZAMPATTI, Emanuela; PREZZA, Miretta; PACILLI, Maria G. Community psychology principles and recent innovations in social policies for children in Italy. Journal of community $\mathcal{E}$ applied social psychology, v. 18, n. 5, p. 512-517, 2008.

RODRIGUES, Fernanda M. G.; PESSOA, Vanete M.; SILVA, Shirley M. Verificação no nível de independência de mobilidade de crianças. Movimentum: revista digital de educação física, v. 2, n.2, p. 1-12, 2007.

UNITED NATIONS CHILDREN'S FUND. THE UNICEF. What is a child friendly citiy? Disponível em: $<$ http:/ / childfriendlycities.org/overview/what-is-achild-friendly-city/> Acesso em: 13 ago. 2013.

VALENTINE, Gill; MCKENDRICK, John Children's outdoor play: exploring parental concerns about children's safety and the changing nature of childhood. Geoforum, v. 28, n. 2, p. 219-235, 1997.

VILLANUEVA, Karen; GILES-CORTI, Billie; BULSARA, Max; TIMPERIO, Anna; MCCORMACK, Gavin; BEESLEY, Bridget; TRAPP, Georgina; MIDDLETON, Nicholas Where do children travel to and what local opportunities are available? The Relationship between neighborhood destinations and children's independent mobility. Environment and Behavior, p. 1-27, 2012.

VISSCHER, Sven de; BIE, Maria Bouverne-de. Recognizing urban public space as a co-educator: children's socialization in Ghent. International journal of urban and regional research, v. 32, n. 3, p. 604-616, 2008. 